\title{
DIGRAPHS AND THE SEMIGROUP OF ALL FUNCTIONS ON A FINITE SET
}

\author{
by PETER M. HIGGINS
}

(Received 25 January 1986; revised 10 September, 1986)

Let $T_{n}$ denote the full transformation semigroup on the finite set $\bar{n}=\{1,2, \ldots, n\}$, that is the set of all mappings from $\bar{n}$ to $\bar{n}$, with function composition as the semigroup operation. In this paper algorithms are introduced to solve equations such as $a x^{m} b=c$ and $a x=x b\left(a, b, c \in T_{n}\right)$, which employ a representation of members of $T_{n}$ as special directed graphs.

Algorithms for equations in $T_{n}$. The object of study here is $T_{n}$, the full transformation semigroup on $\bar{n}=\{1,2, \ldots, n\}$, which means the semigroup of all mappings from $\bar{n}$ to itself under composition. This semigroup is to the theory of algebraic semigroups what the symmetric group (the group of all permutations on a set) is to group theory. It is easy to prove a "Cayley Theorem" to the effect that any semigroup $S$ can be faithfully represented as a subsemigroup of $T_{S^{1}}$, the full transformation semigroup on the set $S^{1}$ (the semigroup $S^{1}$ is the semigroup $S$, with an adjoined identity 1 , if $S$ does not already possess one (see [1])). The full transformation semigroup also enjoys the important property of regularity, meaning that each element $a \in T_{n}$ has an inverse $x$ (not necessarily unique) in the sense that $a=a x a$ and $x=x a x$.

In [3] the author introduced a method for the calculation of all square roots of a given $\alpha \in T_{n}$, the full transformation semigroup on $\bar{n}=\{1,2, \ldots, n\}$, as an alternative to the necessary and sufficient conditions given in [6] for $\alpha$ to be a square. The technique, which relies on a representation of $\alpha$ as a special directed graph, is extended here to furnish algorithms for the solution of the equations:

$$
a x^{m} b=c
$$

and

$$
a x=x b\left(a, b, c \in T_{n}, n \geqslant 1\right) .
$$

The following graph theoretic definitions and results come from [2]. A digraph is said to be weak if it is connected when viewed as a graph. A functional digraph is a weak digraph in which every point has outdegree one. An in-tree is a digraph with a sink (point of outdegree zero), which is a tree when regarded as a graph.

REsULt 1 ([2, Theorem 16.5]). The following are equivalent for a weak digraph $D$.

(1) $D$ is functional.

(2) $D$ has exactly one cycle, the removal of whose arcs results in a digraph in which each component is an in-tree with its sink in the cycle.

(3) $D$ has exactly one cycle $Z$, and removal of any arc of $Z$ results in an in-tree.

Result 2 ([2, Theorem 16.4]). A weak digraph is an in-tree if and only if exactly one point has outdegree zero, and all others have outdegree one.

Glasgow Math. J. 30 (1988) 41-57. 
A tree is said to be rooted, if it has a distinguished point called the root. An in-tree has a natural root in its sink. In what follows a "tree" will always mean an in-tree.

We associate with $\alpha \in T_{n}$ a digraph (which we shall also call $\alpha$ ) on $n$ labelled points, where $i j$ is an arc if $i \alpha=j$. Each point of $\alpha$ has outdegree one, so that the components of $\alpha$ are functional. Each component $A$ of $\alpha$ can be pictured as a cycle $Z_{A}$, together with a family of in-trees rooted at the points of $Z_{A}$. The distance between two points $i$ and $j$ on a digraph, denoted by $d(i, j)$, is the length of any minimal directed path from $i$ to $j$, (if such exists). For a point $u$ of a component $A$ we define $d(u)=0$ if $u \in Z_{A}$, and $d(u)=d(u, 0)$ otherwise, where 0 denotes the sink of the tree of which $u$ is a point. The vertex set of a tree $T$, cycle $Z$ etc. is denoted by $V(T), V(Z)$ etc. The radius $r(T)$ of a tree $T$ is the maximum of $d(u)(u \in V(T))$. It is easy to prove by induction on the radius that the direction of the arcs of a tree are implicitly defined once the sink has been specified. Therefore if we adopt the convention that the cycles of $\alpha \in T_{n}$ are directed anti-clockwise, then the arrows may be deleted from the picture of $\alpha$, with the exception that the picture must provide indication of all cycles of order one (corresponding to fixed points) in order to avoid ambiguity. In the following example this is done by shading the fixed point.

Let $\alpha$ be the member of $T_{20}$ given by

$$
\left(\begin{array}{rrrrrrrrrrrrrrrrrrrr}
1 & 2 & 3 & 4 & 5 & 6 & 7 & 8 & 9 & 10 & 11 & 12 & 13 & 14 & 15 & 16 & 17 & 18 & 19 & 20 \\
2 & 3 & 4 & 5 & 6 & 4 & 2 & 9 & 10 & 11 & 12 & 13 & 14 & 11 & 14 & 17 & 17 & 17 & 17 & 17
\end{array}\right)
$$

Then the corresponding digraph is
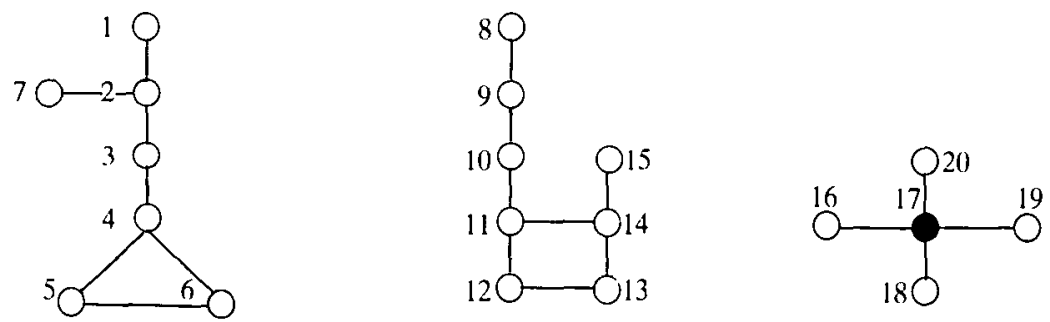

The range of a mapping $\alpha$ will be denoted by $\nabla \alpha$. The rank of $\alpha$ is the order of the range of $\alpha$, denoted by $|\nabla \alpha|$ or rank $\alpha$. The defect of $\alpha \in T_{n}$ is defined as $n-\operatorname{rank} \alpha$. The components of the digraph of $\alpha$ correspond to the orbits of $\alpha$ as defined in [6]; the orbit of $i(i \in \bar{n})$ is $\left\{j \in \bar{n} \mid i \alpha^{r}=j \alpha^{s}\right.$ for some $\left.r, s \in N\right\}$. The stable range of $\alpha$, denoted in [6] by stran $\alpha$, is defined as

$$
\text { stran } \alpha=\left\{i \in \bar{n} \mid i \in \bar{n} \alpha^{k} \text { for all } k=1,2, \ldots,\right\} \text {. }
$$

Note that $\alpha \mid \operatorname{stran} \alpha$ is a permutation, $\operatorname{stran} \alpha=\operatorname{stran} \alpha^{k}$ for all $k=1,2, \ldots$, and that the points of $\operatorname{stran} \alpha$ correspond to the points of the cycles of the digraph of $\alpha$. Observe also that $\operatorname{stran} \alpha=\nabla \alpha^{i}$, where $i$ is the least positive integer such that $\nabla \alpha^{i}=\nabla \alpha^{i+1}$ in the descending chain $\nabla \alpha \supseteq \nabla \alpha^{2} \supseteq \ldots \supseteq \nabla \alpha^{k} \supseteq \ldots$. It follows that $\alpha$ partitions $\bar{n}$ into two sets corresponding to stran $\alpha$ and its complement, and that this partition is invariant under the taking of powers, and thus the taking of $m$ th roots also. This key observation will allow 
the problem of solution of equations of type (1) to be split into two constructions corresponding to the classes of this partition.

The digraph representation of members of $T_{n}$ has been employed extensively by Howie. In [4] Howie showed that the subsemigroup of $T_{n}$ generated by $E$, the set of all idempotents of $T_{n}$ of defect one, is the semigroup $S$ of all "singular" mappings:

$$
\langle E\rangle=S=\left\{\alpha \in T_{n} \mid \text { rank } \alpha<n\right\} .
$$

(Note that $T_{n}$ is the disjoint union of $S$ and the symmetric group on $\bar{n}$.) Later in [5], Howie found the least integer $k$ such that $\alpha \in E^{k}$ for a given $\alpha \in T_{n}$. The answer can be expressed succinctly in terms of features of the digraph of $\alpha$ :

$$
k=n-c(\alpha)+f(\alpha),
$$

where $f(\alpha)$ is the number of fixed points of $\alpha$, and $c(\alpha)$ is the number of cyclic orbits of $\alpha$. (An orbit is cyclic if it is a cycle of order at least two.)

We begin our attack on equation (1) with the special case where $a=b$ equals the identity map. In other words we seek all the $m$ th roots of $\alpha \in T_{n}$. First we observe that it is enough to solve

$$
x^{p}=\alpha,
$$

where $p$ is prime. For a subset of $S$ of $T_{n}$ define

$$
S^{1 / m}=\bigcup_{\alpha \in S} \alpha^{1 / m}, \quad \text { where } \quad \alpha^{1 / m}=\left\{\beta \mid \beta^{m}=\alpha\right\} .
$$

Since

$$
\left(\alpha^{1 / k}\right)^{1 / m}=\left(\alpha^{1 / m}\right)^{1 / k}=\alpha^{1 / m k},
$$

it follows that $\alpha^{1 / m}$ can eventually be calculated provided $\alpha^{1 / p}$ can be found for any prime $p$.

After the fashion of [3], we approach our problem by first identifying features of a typical $p$ th power in $T_{n}$. To this end, let $\alpha \in T_{n}$ and let $A$ be a component of $\alpha$. By the order of $A$, denoted by $o(A)$, we shall mean the order of the cycle, $Z_{A}$ of $A$. We may also regard $A$ as a mapping whose domain and codomain is the set of points of $A$; whereupon we may speak of $A^{k}$ as the digraph corresponding to the $k$ th power of the map associated with $A$, while $A^{1 / k}$ means the set of all $k$ th roots of $A$.

The map $A^{p}$ has two fundamentally different forms according as $p$ does or does not divide $o(A)$.

LEMMA 3. The map $A^{p}$ has one component or $p$ components according as $o(A) \not \equiv 0(\bmod p)$ or $o(A) \equiv 0(\bmod p)$.

Proof. Suppose that $o(A)=k \not \equiv 0(\bmod p)$. Take $i \in V\left(Z_{A}\right)$. Then the points $i \alpha^{p}$, $i \alpha^{2 p}, \ldots, i \alpha^{k p}$ are all distinct, for if $i \alpha^{r p}=i \alpha^{s p}(1 \leq r, s \leq k)$ then $r p \equiv s p(\bmod k)$, whence $r \equiv s(\bmod k) \quad$ as $\quad(p, k)=1$. Hence $r=s$. We obtain $V\left(Z_{A}\right)=\operatorname{stran} A=\operatorname{stran} A^{p}=$ $\left\{i \alpha^{p}, i \alpha^{2 p}, \ldots, i \alpha^{k p}\right\}$, and since these points are obviously in the same orbit in $A^{p}$, it follows that $A^{p}$ has but one component. 
On the other hand suppose that $o(A)=k p$ for some $k \geq 1$. Then for any $i \in Z_{A}, k$ is the least positive integer such that $i=i \alpha^{p k}$. Hence stran $A^{p}=V\left(Z_{A}\right)$ is partitioned into $o(A) / k=p$ cycles, each of order $k$, corresponding to the $p$ distinct components of $A^{p}$.

Next we investigate the behaviour of the points of a tree $T$, rooted on the cycle of $A$, when $A$ is raised to the $p$ th power.

Lemma 4. Let $T$ be a tree rooted on the cycle $Z_{A}$ of $A$. Denote $r(T)(\bmod p)$ by $k$. The non-root points of $T$ form the non-root points of $p$ trees $\left(T_{1}, T_{2}, \ldots, T_{p}=T_{0}\right)$ in $A^{p}$ that we shall call the $p$ th order offspring of $T$. Furthermore, $r\left(T_{1}\right)=r\left(T_{2}\right)=\ldots=r\left(T_{k}\right)=$ $r\left(T_{k+1}\right)+1=r\left(T_{k+2}\right)+1=\ldots=r\left(T_{p}\right)+1$ if $k \neq 0$, and all radii are equal if $k=0$. If $p \mid O(A)$ then the $T_{1}, \ldots, T_{p}$ are rooted one on each of the $p$ distinct cycles of the components of $A^{p}$. If $p \nmid o(A)$ then $T_{1}, \ldots, T_{p}$ are rooted on the unique cycle of $A^{p}$. Regard the points of $Z_{A}$ in anti-clockwise order as the integers modulo o $(A)$ beginning with 0 , the root of $T$. Then the roots of $T_{1}, T_{2}, \ldots, T_{p}$ are respectively $p-1, p-2, \ldots, 0$.

Before formally verifying this lemma we illustrate the behaviour described by means of a pair of examples corresponding to the case $p \mid o(A)$ and $p \nmid o(A)$ respectively. In both examples $p=3$ and two vertices are labelled by the same letter if they occur in the same tree in the offspring triple. In the first case $A$ consists of a single component of order three.

EXAMPLE 1.

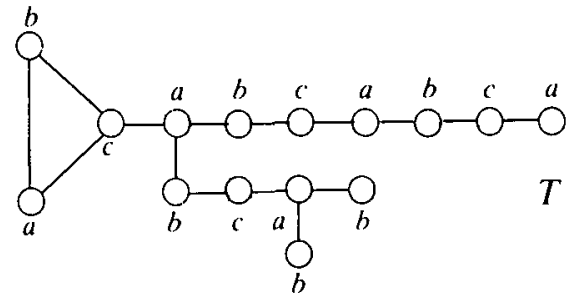

We can think of $A$ as a member of $T_{15}$ with the points labelled by the integers 1 to 15 in some manner. Consider $A^{3}$. The digraph of $A^{3}$ is thus
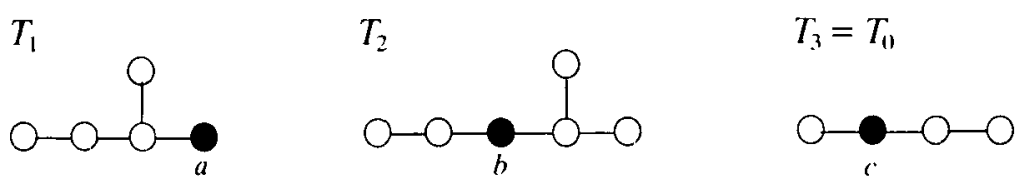

where the direction of arcs is understood to be towards shaded points, and the points in the stable range still carry their original labels. The second example involves a single component of order four, but in all other respects resembles our first digraph.

EXAMPLE 2.

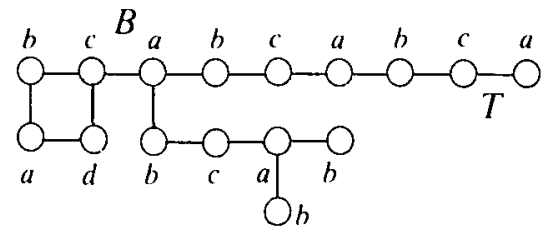


Since $p \nmid o(B)$ it is easy to see that $B^{3}$ has but one component, and it is also of order four. The tree of $B$ splits into an "offspring triple" exactly as in the first example.

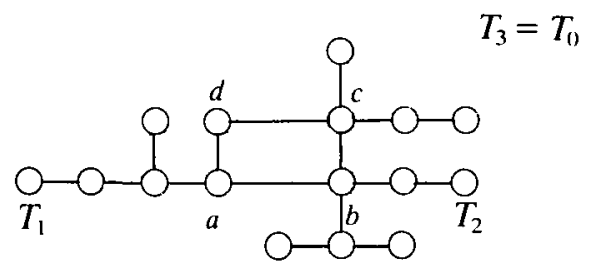

In both examples $r(T)=7$ and $k=r(T)(\bmod p)=7(\bmod 3)=1$.

In accordance with the lemma we have $r\left(T_{1}\right)=r\left(T_{2}\right)+1=r\left(T_{3}\right)+1=3$. Note also that in both examples the positioning of the roots of the offspring triple is consistent with the statement of the lemma: the roots of the triple in order are $a, b, c$, which correspond to the order that occurs in the inverse of the cycles of $A$ and $B$. Also the root of $T_{3}=T_{0}$ is that of $T$.

These examples serve to indicate that our problem of reconstructing $p$ th roots will split into the related tasks of building root cycles of the given collection of cycles, together with parent trees of collections of offspring trees, and combining the two in a consistent manner.

Proof of Lemma 4. The non-root points of $T$ are not members of stran $A$, and so are not members of stran $A^{p}$. It follows that the non-root points of $T$ form the vertices of a number of trees in $A^{p}$. Observe that for points $u$ and $v$ of $T, d(u, 0) \equiv d(v, 0) \equiv i(\bmod p)$ if and only if $u$ and $v$ are points which appear on a tree of $A^{p}$ rooted at $p-i$. Hence $T$ gives rise to $p$ trees $\left(T_{1}, T_{2}, \ldots, T_{p}\right)$ in $A^{p}$, with $u, v \in V\left(T_{i}\right)$ if and only if $d(u, 0) \equiv d(v, 0) \equiv i(\bmod p)$ and $T_{i}$ is rooted at $p-i, i=1,2, \ldots, p$. (Note that it is possible that some of the $T_{i}$ may have no non-root points.)

Moreover $r\left(T_{i}\right)$ equals the number of vertices of $V\left(T_{i}\right)$ on any maximal path of $T$, whence $r\left(T_{1}\right)=r\left(T_{2}\right)=\ldots=r\left(T_{k}\right)=r\left(T_{k+1}\right)+1=r\left(T_{k+2}\right)+1=\ldots=r\left(T_{p}\right)+1$, unless $k=0$ whereupon all the radii are equal. The remaining part of the statement follows from the proof of Lemma 3.

We may now give an algorithm for constructing the $p$ th roots of $\alpha \in T_{n}$, up to the problem of constructing all the parent trees $T$ of a given offspring $p$-tuple $\left(T_{1}, T_{2}, \ldots, T_{p}\right)$.

Again it is perhaps more instructive to work from the particular to the general. Suppose we were given $\alpha \in T_{15}$ whose digraph had the form of $A^{3}$ in our first example, and were asked to construct all its cube roots. We might begin by examining the stable range and asking ourselves, what cycle structures give, upon cubing, a triple of one-cycles? Since any cube root has the same three point set as stable range, the stable range of any cube root must consist of a three-cycle, a two-cycle and one fixed point, or three fixed points. Lemma 3 precludes the second possibility (the cubing of a 2-cycle results in a 2-cycle). A cube root with three fixed points is also an impossibility as a little 
careful thought reveals. Suppose that $\beta^{3}=\alpha$ and that $\operatorname{stran} \beta$ consisted of three one-cycles. Let $T$ be a tree of $\beta$ with sink the fixed point $a$ say. In $\beta^{3}, T$ corresponds to an offspring triple of trees $\left(T_{1}, T_{2}, T_{3}\right)$ rooted on $a$, and the radii of these trees differ by at most one. Obviously the trees rooted on $a$ in $A^{3}$ cannot be gathered into such triples (and the same applies to $b$ and $c$ ). Hence there is no such $\beta$. Therefore the stable range of any cube root of $A^{3}$ must be one of two possible 3-cycles. Which ever 3-cycle is chosen, the trees rooted around the fixed points of $A^{3}$ can be listed in offspring triples. For each such triple a parent tree must be constructed and rooted at an appropriate position on the root cycle. There are two possible complications that we should note. It is possible for an offspring triple to contain one or two trivial trees of radius zero (the non-trivial trees in the triple would then have radius one). The second difficulty is apparent upon examination of the second (or third) component of $A^{3}$ with fixed point labelled by $b$. The tree $T_{2}$ could equally be regarded as a pair of trees, as the in-degree of the sink $b$ is two. These possibilities must be remembered when one is required to construct all $p$ th roots of a given mapping. Nevertheless in this case it is clear that the only possible way of collecting the trees of $A^{3}$ into a collection of triples in which the radius difference within each triple is at most one is to have one triple consisting of $T_{1}, T_{2}$ and $T_{3}$ in some order. Furthermore the first entry in the triple must be $T_{1}$, because its radius exceeds that of $T_{2}$ and $T_{3}$. This leaves us two choices, and so to be definite let us select the triple $\left(T_{1}, T_{2}, T_{3}=T_{0}\right)$. The next task is to construct a parent tree $T$, and place the sink of $T$ at an appropriate point on the chosen root cycle to ensure that we have a cube root of $A^{3}$. We know in this case of course, this is possible in at least one way because we possess one cube root in $A$ itself. A discussion of an algorithm for the construction of all parent trees of a given triple, or more generally of a given $p$-tuple, will be temporarily postponed. Let us fix attention on the known parent $T$ of $\left(T_{1}, T_{2}, T_{3}\right)$. The last statement in Lemma 4 shows that the "root cycle" is determined by the ordering of the offspring triple: in this case the roots of $\left(T_{1}, T_{2}, T_{3}\right)$, regarded as a cycle, is the inverse of the root cycle. Hence the root cycle is $(a b c)^{-1}=(c b a)$. The root of the parent tree $T$ is $c$, the root of $T_{3}=T_{0}$. Similarly if the offspring $\left(T_{1}, T_{3}, T_{2}\right)$ is chosen we can construct one cube root for each parent tree (but, as we shall show later, there are none), $T^{\prime}$ of this triple: the parent cycle in this case would be $(a b c)$ and the root of $T^{\prime}$ would necessarily be $b$, the root of $T_{2}$.

Next consider the problem of finding the cube roots of the mapping given by $B^{3}$. Since $B^{3}$ has just one cycle, the same is true of all its cube roots. Indeed it is clear that the stable range of any cube root is the unique cube root of the given cycle, $(a b c d)$, which is $(d c b a)$. Once more the trees must be listed in offspring triples. For each parent $T$ of the triple $\left(T_{1}, T_{2}, T_{3}\right)$ there results a cube root, with the root of $T$ placed at $c$. As mentioned above, the other conceivable ordering of the offspring triple, $\left(T_{1}, T_{3}, T_{2}\right)$ has no parent. However that there are no more cube roots of $B^{3}$ can be deduced without knowing this, because the positioning of the roots of the trees around the cycle of $B^{3}$ is inconsistent with these trees forming the offspring triple $\left(T_{1}, T_{3}, T_{2}\right)$; the root cycle is $(d c b a)$, whence it follows from the last statement of Lemma 4 that order of the roots of the offspring triple must be three consecutive members of the inverse cycle $(a b c d)$, but the roots of $\left(T_{1}, T_{3}, T_{2}\right)$ are in order $a, c, b$. 
The problem of constructing $p$ th roots falls naturally into two parts: the problem of reconstructing the stable range of the root, and the problem of building parents of $p$ th order offspring, while both procedures must be carried out in a manner consistent with the positioning of the offspring trees. The problem of recognizing whether or not a given $p$-tuple of trees has its roots positioned in a consistent fashion is dealt with in the following algorithm which solves the equation $x^{p}=\alpha$ modulo the construction of all parents of a given offspring $p$-tuple.

The Root Algorithm. Let $\alpha \in T_{n}$. Then all the $p$ th roots of $\alpha$ are formed as follows: collect the components of $\alpha$ into $p$-tuples $\left(A_{1}, A_{2}, \ldots, A_{p}\right)$ where either

(i) $A_{1}=A_{2}=\ldots=A_{p}=A$ say, $o(A) \not \equiv 0(\bmod p)$ and the trees of $Z_{A}$ are collected in offspring $p$-tuples $\left(T_{1}, T_{2}, \ldots, T_{p}\right)$ (every tree appearing exactly once) where the length of the path between the roots of $T_{i+1}$ and $T_{i}(i=1, \ldots, p-1)$ is $k$, where $k$ is the least positive integer such that $k p \equiv 1(\bmod o(A))$; (we call such a positioning of the roots of the $T_{i}$ consistent); or

(ii) the $A_{i}$ are all distinct, of the same order, and the trees rooted on the $Z_{A_{i}}$ are gathered in offspring $p$-tuples (every tree appearing just once) $\left(T_{1}, T_{2}, \ldots, T_{p}\right)$ with one tree in each $p$-tuple from each $A_{i}$.

In case (i) there is a unique $p$ th root $Z_{A}^{\prime}$ of the cycle $Z_{A}$. A $p$ th root of $A$ results by constructing a parent tree $T$ for each $p$-tuple $\left(T_{1}, T_{2}, \ldots, T_{p}\right)$ with root on $Z_{A}^{\prime}$ as the root of $T_{p}$, and all $p$ th roots of $A$ arise in this way.

In case (ii) each $p$-tuple offspring $\left(T_{1}, T_{2}, \ldots, T_{p}=T_{0}\right)$ defines a unique $p$ th root cycle of order $o\left(A_{i}\right) p$, for which the roots of $T_{1}, \ldots, T_{p}$ appear consecutively clockwise. It is thus necessary that the offspring $p$-tuples of the $A_{i}$ be rooted consistently, meaning that each $p$-tuple defines the same $p$ th root cycle. If this is the case, a $p$ th root of $\left(A_{1}, A_{2}, \ldots, A_{p}\right)$ results by constructing the $p$ th root cycle, and rooting one parent tree $T$ of each $\left(T_{1}, T_{2}, \ldots, T_{p}\right)$ at the root of $T_{p}$ on the $p$ th root cycle. What is more, all $p$ th roots of $\left(A_{1}, A_{2}, \ldots, A_{p}\right)$ arise in this way.

Most of the above has been shown in the foregoing analysis. The first detail which has not been established is that concerning the path length between the roots of $T_{i+1}$ and $T_{i}$ in case (i). To this end suppose that $\beta^{p}=\alpha$, and that $B$ is a component of $\beta$ such that $B^{p}=A$. Any tree $T$ of $B$ gives rise to an offspring $p$-tuple $\left(T_{1}, T_{2}, \ldots, T_{p}\right)$ where the roots of $T_{i+1}$ and $T_{i}$ are points which are anti-clockwise adjacent in $Z_{B}$. Now $V\left(Z_{B}\right)=V\left(Z_{A}\right)$ and so the length of the path between these roots in $Z_{A}$ will be $k$, where $k$ is least such that $k p \equiv 1(\bmod o(A))$. Continuing in case (i), let $Z_{A}$ be given by $(12 \ldots r)$ where $p \nmid r$. Then $Z_{A}$ has as its unique $p$ th root cycle the cycle in which $i(1 \leq i \leq r)$ is followed by $i+k(\bmod r)$, where $k$ is the unique solution to $k p \equiv 1(\bmod r)$, because this is precisely the condition that $i+1(\bmod r)$ occurs exactly $p$ places after $i$ in the $p$ th root cycle.

Finally in case (ii), given the $Z_{A_{i}}(1 \leq i \leq p)$, there is in general more than one $p$ th root cycle $Z_{A}\left(o(A)^{p-1}\right.$ such cycles in fact). Note that in $Z_{A}$ any $p$ consecutive members form a transversal of the $Z_{A_{i}}$, and indeed once a transversal of the $Z_{A_{i}}$ is chosen, there is 
exactly one way to complete the cycle $Z_{A}$. However, the roots of $\left(T_{p}, T_{p-1}, \ldots, T_{1}\right)$ will form $p$ consecutive points in $Z(A)$, and so there is a unique $p$ th root cycle of the $Z_{A_{i}}$ which is consistent with the positioning of the roots of the $T_{i}$. This establishes the remark on consistency.

We next seek an algorithm for the construction of all parent trees $T$ (if any) for a given $p$-tuple of trees $\left(T_{1}, T_{2}, \ldots, T_{p}\right)$. We have thus far considered such a $p$-tuple as a subgraph of the digraph of some $\alpha \in T_{n}$. It now becomes convenient to consider the $p$-tuple $\left(T_{1}, T_{2}, \ldots, T_{p}=T_{0}\right)$ of trees with disjoint sets of non-root points without reference to any particular $\alpha$. A parent tree $T$ is then considered to be a tree which has as non-root points the non-root points of $T_{1}, T_{2}, \ldots, T_{p}$ and the same root as $T_{p}$, with the additional properties that for two vertices $u, v \in V(T), d(u, v) \equiv 0(\bmod p)$ if and only if $u, v \in V\left(T_{i}\right)$ for some $i=1,2, \ldots, p$, and for such a pair of vertices $d(u, 0)>d(v, 0)$ if and only if $d\left(u, 0_{i}\right)>d\left(v, 0_{i}\right)$, (where 0 and $0_{i}$ are the sinks of $T$ and of $T_{i}$ respectively).

We shall picture this parent $T$ as rooted on some unspecified cycle $Z_{A}$ of some component $A$ of a mapping $\alpha$. The offspring of $T$ which results when $\alpha$ is raised to the $p$ th power is then indeed $\left(T_{1}, T_{2}, \ldots, T_{p}\right)$. The corresponding roots of the offspring $p$-tuple, $0_{1}, 0_{2}, \ldots, 0_{p}=0$ will be points of the unspecified cycle. They will be distinct if and only if $\left|Z_{A}\right| \geq p$. However this is of no consequence in the following parent tree algorithm.

Suppose that a parent tree $T$ exists. Take a maximal path $P$ of $T$ from an endpoint $u$ of $T$ to the sink 0 , and label the points of $P$ by $t, t-1, t-2, \ldots, 0$ where $d(u, 0)=t \geq 1$. The path $P$ corresponds to maximal paths $P_{1}, P_{2}, \ldots, P_{p}=P_{0}$ of $T_{1}, T_{2}, \ldots, T_{p}$ respectively, such that $\left|P_{1}\right|=\left|P_{2}\right|=\ldots=\left|P_{k}\right|=\left|P_{k+1}\right|+1=\ldots=\left|P_{p}\right|+1$ where $t \equiv k(\bmod p)$ and $\left|P_{j}\right|$ denotes the length of the path $P_{j}($ note here we take $k=p$ if $t \equiv 0(\bmod p)$ : whereupon the statement also holds in the case where all lengths are equal).

We shall say that a point $u$ of $P_{i}$ is at position $j$ if $d\left(u, 0_{i}\right)=j$. Now consider a tree $T^{\prime}$ rooted at position $l=r p+j$ on $P(0 \leq j \leq p-1)$. The sub-tree $T^{\prime}$ of $T$ gives rise to a $p$-tuple of its own, $\left(T_{1}^{\prime}, T_{2}^{\prime}, \ldots, T_{p}^{\prime}=T_{0}^{\prime}\right)$ rooted on the paths $P_{j+1}, P_{j+2}, \ldots, P_{p}, P_{1}$, $P_{2}, \ldots, P_{j}$ respectively. This follows as $u \in V\left(T_{i}^{\prime}\right)$ if and only if $d(u, l) \equiv i(\bmod p) \Leftrightarrow$ $d(u, 0) \equiv i+r p+j(\bmod p) \equiv j+i(\bmod p)$. Furthermore, $T_{1}^{\prime}, T_{2}^{\prime}, \ldots, T_{p-j}^{\prime}$ are rooted at position $r$ on $P_{j+1}, P_{j+2}, \ldots, P_{p}$ respectively, while $T_{p-j+1}^{\prime}, T_{p-j+2}^{\prime}, \ldots, T_{p}^{\prime}$ are rooted at position $r+1$ on $P_{1}, P_{2}, \ldots, P_{j}$ respectively. We shall also call $\left(T_{1}^{\prime}, T_{2}^{\prime}, \ldots, T_{p}^{\prime}=T_{0}^{\prime}\right)$ a consistently rooted offspring $p$-tuple of $T^{\prime}$.

Again let us illustrate these observations by means of the following example.

EXAmple 3.

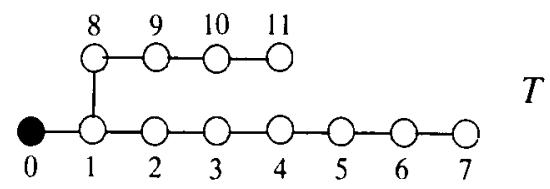

Obviously $T$ has a unique maximal path $P$. The third order offspring of $T$ are $\left(T_{1}, T_{2}, T_{3}\right)$ : 


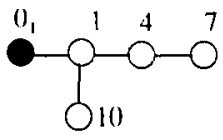

$T_{1}$

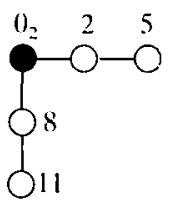

$T_{2}$

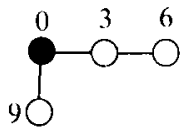

$T_{3}$

The maximal paths $P_{1}, P_{2}, P_{3}$ corresponding to $P$ are

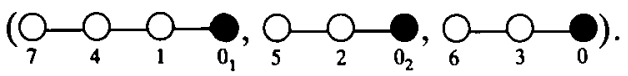

We have $t=7 \equiv 1(\bmod 3)$, hence $k=1$, and in accord with this we have $3=\left|P_{1}\right|=$

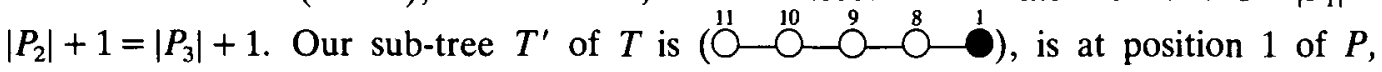
and $1=0.3+1$, giving $r=0, j=1$ in this case, and $\left(T_{1}^{\prime}, T_{2}^{\prime}, T_{3}^{\prime}\right)=\left(0_{11}^{0}-O_{8}-O_{2}\right.$, $\left.\bigcirc_{9}-O_{0}, \bigcirc_{10}\right)$. Hence we find that $T_{1}^{\prime}, T_{2}^{\prime}$ and $T_{3}^{\prime}$ are rooted on $P_{2}, P_{3}$ and $P_{1}$ respectively with $T_{1}^{\prime}$ and $T_{2}^{\prime}$ rooted at position 0 while $T_{3}^{\prime}$ is rooted at position 1 , in accord with our general description.

These observations allow construction of all parent trees of a consistently rooted $p$-tuple $\left(T_{1}, T_{2}, \ldots, T_{p}\right)$ in the digraph of $\alpha \in T_{n}$.

The Parent Tree Algorithm. Let $\left(T_{1}, T_{2}, \ldots, T_{p}\right)$ be a consistently rooted $p$-tuple of trees occurring in the digraph of $\alpha \in T_{n}$. The following construction yields all parent trees of $\left(T_{1}, T_{2}, \ldots, T_{p}\right)$.

Assume inductively that we may construct all parent trees of any non-trivial, consistently rooted $p$-tuple $\left(T_{1}^{\prime}, T_{2}^{\prime}, \ldots, T_{p}^{\prime}\right)$ for which the total number of points is less than the total number of points of $\left(T_{1}, T_{2}, \ldots, T_{p}\right)$. (There is no difficulty starting this inductive argument, for the $p$-tuple $\left(\underset{1}{(-O}, O_{0}, \ldots, 0_{0}, \ldots\right)$ has a unique parent tree in $\left.\left(\stackrel{1}{O^{0}} 0_{0}\right)\right)$. Select a $p$-tuple $\left(P_{1}, P_{2}, \ldots, P_{p}\right)$ where $P_{j}$ is a maximal path to the root $0_{j}$ of $T_{j}$ such that $\left|P_{1}\right|=\left|P_{2}\right|=\ldots=\left|P_{k}\right|=\left|P_{k+1}\right|+1=\left|P_{k+2}\right|+1=\ldots=\left|P_{p}\right|+1$ for some $k \epsilon$ $\{1,2, \ldots, p\}$. Furthermore do this in such a fashion that the sub-trees occurring on the paths $P_{1}, P_{2}, \ldots, P_{p}$ can themselves be listed in consistently rooted offspring $p$-tuples $\left(T_{1}^{\prime}, T_{2}^{\prime}, \ldots, T_{p}^{\prime}\right)$ meaning that $T_{1}^{\prime}, T_{2}^{\prime}, \ldots, T_{p}^{\prime}$ occur on $P_{j+1}, P_{j+2}, \ldots, P_{p}, P_{1}, \ldots, P_{j}$ respectively (for some $0 \leq j \leq p-1$ ) with $T_{1}^{\prime}, T_{2}^{\prime}, \ldots, T_{p-j}^{\prime}$ rooted at position $r$ for some $r \geq 0$, and $T_{p-j+1}^{\prime}, T_{p-j+2}^{\prime}, \ldots, T_{p}^{\prime}$ rooted at position $r+1$.

Next, construct a path $P$ from $\left(P_{1}, P_{2}, \ldots, P_{p}\right)$ as follows: relabelling the points of $P_{i}$ from the sink as $0_{i 0}, 0_{i 1}, 0_{i 2}, \ldots$, the points of $P$ from the sink $0_{p 0}$ are: $0_{p 0}, 0_{11}$, $0_{21}, \ldots, 0_{p 1}, 0_{12}, 0_{22}, \ldots, 0_{p 2}, \ldots, 0_{1 t}, 0_{2 t}, \ldots, 0_{k t}$, where $t=\left|P_{1}\right|=\left|P_{2}\right|=\ldots=\left|P_{k}\right|$. For each $p$-tuple $\left(T_{1}^{\prime}, T_{2}^{\prime}, \ldots, T_{p}^{\prime}\right)$ construct a parent tree $T^{\prime}$ rooted at position $r p+j$ on $P$. The tree $T$ so constructed is a parent of $\left(T_{1}, T_{2}, \ldots, T_{p}\right)$ and all parent trees are constructed in this way. 
As an example to illustrate this algorithm let us find all parent trees of the triple $\left(T_{1}, T_{2}, T_{3}\right)$ of Example 1:
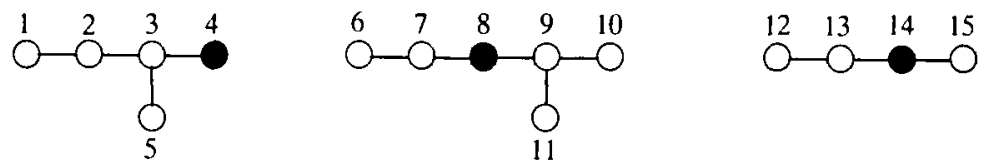

There is just one choice of $P_{1}$ and $P_{3}$ and three choices for $P_{2}$. Let's begin with

$$
\left(P_{1}, P_{2}, P_{3}\right)=(\stackrel{1}{O}-\stackrel{2}{O}-\stackrel{3}{O}-\stackrel{4}{\circ}, \stackrel{6}{\circ}-\stackrel{7}{O}-\stackrel{8}{\circ}, \stackrel{12}{\circ}-\stackrel{13}{O}-\stackrel{14}{\circ}) \text {. }
$$

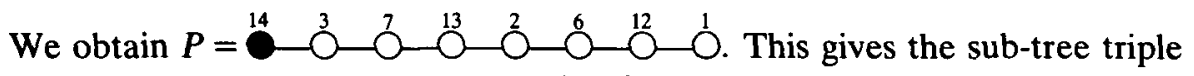

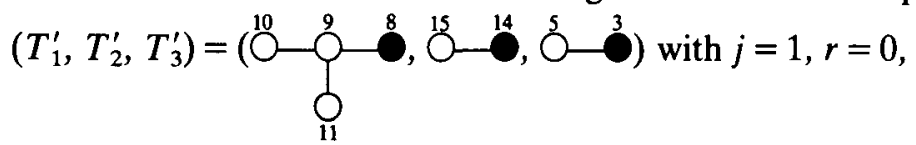

whence $T_{1}^{\prime}, T_{2}^{\prime}$ are positioned at 0 and $T_{3}^{\prime}$ positioned at 1 . Repeating the procedure we get

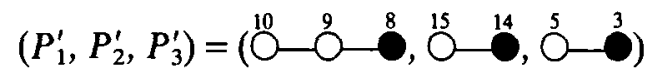

and thus

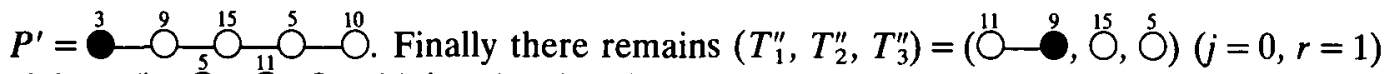
giving $P^{\prime \prime}=-\mathrm{O}$. Combining $P, P^{\prime}$ and $P^{\prime \prime}$ we get a parent tree

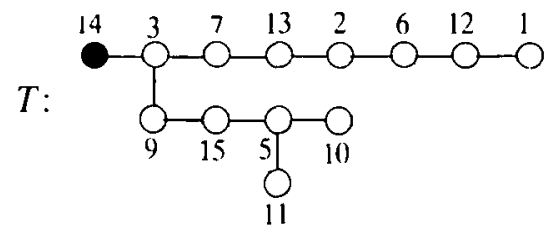

Attaching $T$ to the cycle (1484) in effect gives us our original cube root $A$ of $A^{3}$. There are another two which can be constructed by selecting different choices for $P_{2}$.

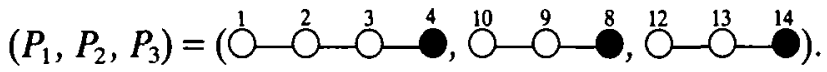

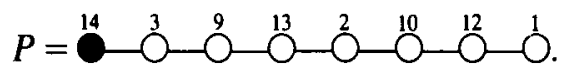

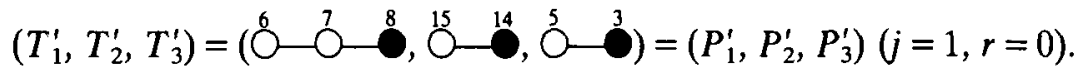

$$
\begin{aligned}
& P^{\prime}=3 \\
& \left(T_{1}^{\prime \prime}, T_{2}^{\prime \prime}, T_{3}^{\prime \prime}\right)=(\stackrel{11}{\bigcirc}-\stackrel{9}{\circ}, \stackrel{13}{\bigcirc}, \stackrel{2}{\bigcirc})=\left(P_{1}^{\prime \prime}, P_{2}^{\prime \prime}, P_{3}^{\prime \prime}\right)(j=r=1) \text {. } \\
& P^{\prime \prime}=\stackrel{2}{-11} \text {. Combining, we get another parent tree }
\end{aligned}
$$




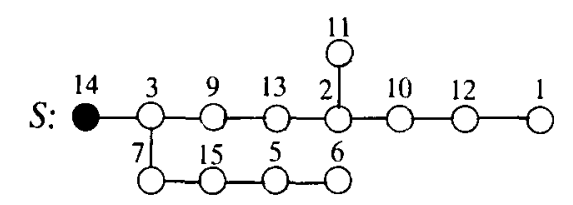

The third choice for $P_{2}$ is $\mathrm{O}^{11} O^{9}-8$. This will simply interchange the roles played by the points 10 and 11 in the construction, thus giving a third parent $R$, identical to $S$ except that the labels 10 and 11 are swapped.

The other conceivable offspring in $A^{3}$ is $\left(T_{1}, T_{3}, T_{2}\right)$. However the algorithm to construct parents breaks down when applied. We take $\left(P_{1}, P_{2}, P_{3}\right)=\left({ }_{0}^{4}-\Omega_{0}^{3}-S_{0}^{1}\right.$, ${ }^{14} \mathrm{I}^{13}-P_{3}$ ), where there are three possible choices for $P_{3}$. However, regardless of which $P_{3}$ is chosen, $P_{3}$ has a radius two subtree positioned at zero, which must be the first entry of a triple of the form $\left(T_{1}^{\prime}, T_{2}^{\prime}, T_{3}^{\prime}\right)$ with $T_{1}^{\prime}$ on $P_{3}, T_{2}^{\prime}$ on $P_{1}, T_{3}^{\prime}$ on $P_{2}$ and $T_{1}^{\prime}$ rooted at $0, T_{2}^{\prime}$ and $T_{3}^{\prime}$ rooted at 1 (as $j=2$ ). Plainly, there is no suitable $T_{3}^{\prime}$ on $P_{2}$.

Therefore we have in effect found all three cube roots of our mapping $A^{3}$ of Example 1.

It is now possible to solve our equation (1) $a x^{m} b=c\left(a, b, c \in T_{n}\right)$. First consider the equation $a y b=c$. The map $\alpha$ is a solution if and only if $(i a) \alpha \in(i c) b^{-1}$ for all $i \in \bar{n}$. All such $\alpha$ can easily be found. We then solve the original equation $a x^{m} b=c$ by calculating $\left\{y \in T_{n} \mid a y b=c\right\}^{1 / m}$ using the above algorithms repeatedly.

As an example, let us find all cube roots of $\alpha \in T_{32}$ :

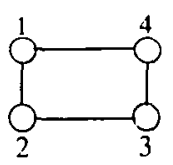

$A$

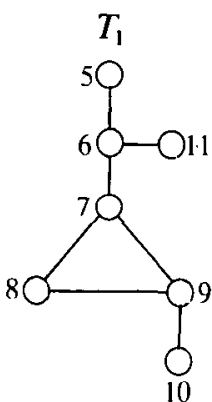

$B$

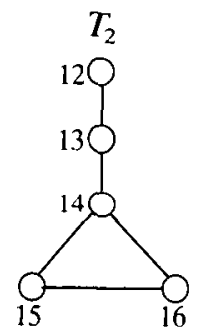

C

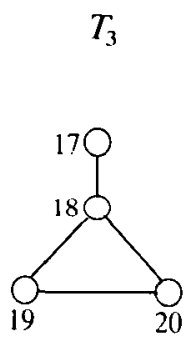

$D$

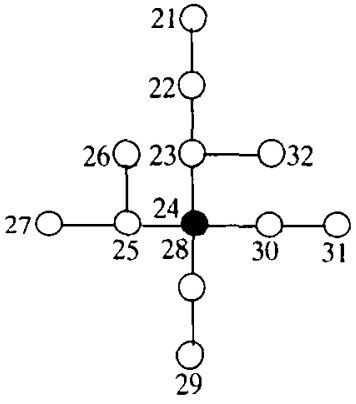

$E$

The only listing of the components which might be consistent with The Root Algorithm is $(A, A, A),(B, C, D)$ and $(E, E, E)$. (The ordering of the components within the triple $(B, C, D)$ is of no importance: any ordering leads to the same analysis). The cyclic component $A$ has a unique cube root in (1432). Next we consider $(B, C, D)$, and we try to form a parent tree for the triple $\left(T_{1}, T_{2}, T_{3}\right)$. We take $P_{1}=\mathrm{O}^{5}-\mathrm{O}^{6}-P_{2}=P^{12} \mathrm{O}^{13}-\mathrm{C}^{14}$, $P_{3}=\mathrm{O}^{18}$. There is only one sub-tree on $P_{1}, P_{2}$ or $P_{3}$, the tree $\mathrm{O}^{11}$ occurring at the 
second vertex of $T_{1}$. This can be associated with the trivial trees at the points 13 and 17 of $P_{2}$ and $P_{3}$ respectively, to give a triple $\left(T_{1}^{\prime}, T_{2}^{\prime}, T_{3}^{\prime}\right)=(\stackrel{11}{-}-6, \stackrel{13}{\bigcirc}, \stackrel{17}{O})$ in accordance with the Parent Tree Algorithm $(p=3, r=1, j=0, k=2)$. This gives the parent tree $T$

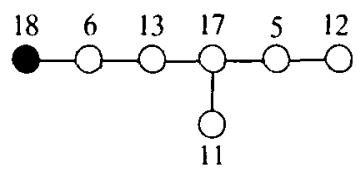

The cube root of the triple of cycles $\left(Z_{B}, Z_{C}, Z_{D}\right)$ defined by this offspring triple is (18 $14 \quad 7 \quad 1915 \quad 82016$ 9). The tree $\mathrm{O}^{10}=$ is easily accommodated (clearly 10 must be mapped to 20 in the cube root) but formally we list the triple $(\stackrel{10}{0}, \stackrel{16}{\circ}, \stackrel{20}{0})$ $(p=3, k=1)$, and form the parent tree $\mathrm{O}^{20}$ with consistent positioning of the roots (i.e. this triple defines the same parent cycle). The other possible choice for $P_{1}$ is 11-7 ${ }^{6}$; this leads to a cube root in which 12 is mapped to 11 . Also there is another possible listing of the trees, viz. $\left(T_{2}, T_{1}, T_{3}\right)$, which leads to two distinct cube roots of $(B, C, D)$, giving four in all.

Finally we deal with the component $E$. One cannot argue that since there are four trees rooted on the fixed point, they cannot be listed in offspring triples, as a tree may have more than one branch at its root. If a cube root exists, the trees of $E$ must be listed in a triple $\left(T_{1}, T_{2}, T_{3}\right)$ with exactly one of the trees with two branches at the root. Also $T_{1}$ must contain the topmost tree (containing the point 21), as it has a maximal path whose length exceeds any other. It is also true that one of $T_{2}, T_{3}$ must be The alternative would see $\mathrm{O}^{29} \mathrm{O}^{28}$ or $\mathrm{O}^{31}-\mathrm{SO}^{24}$ as one of $T_{2}, T_{3}$; to be definite let us suppose it is $\mathrm{O}_{-2}^{28}$. However, then the member of $\left(T_{1}, T_{2}, T_{3}\right)$ with two root branches would have a subtree of radius two at its root, which could not be listed in a suitable subtree triple $\left(T_{1}^{\prime}, T_{2}^{\prime}, T_{3}^{\prime}\right)$ as ${ }^{29}-{ }^{28}-24$ has no non-trivial subtrees. Hence we attempt to construct a parent for $\left(T_{1}, T_{2}, T_{3}\right)$ as given below:
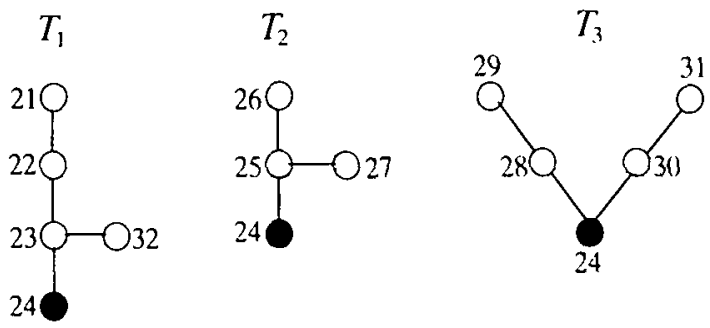

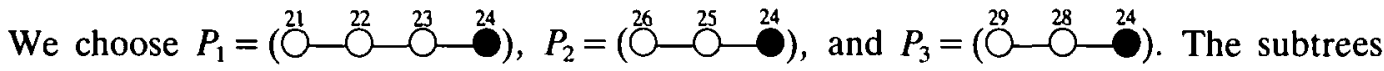
of $T_{1}, T_{2}, T_{3}$ can then be accommodated in just one way consistent with the criteria of the

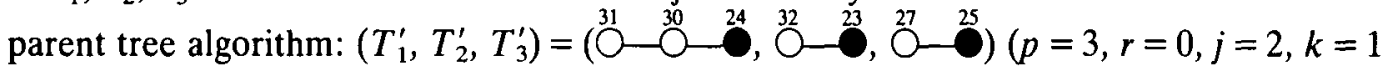


and $r p+j=2$ ), as the radius of $T_{1}^{\prime}$ exceeds the radii of the other two subtrees. This allows us to construct a parent tree $T$ :

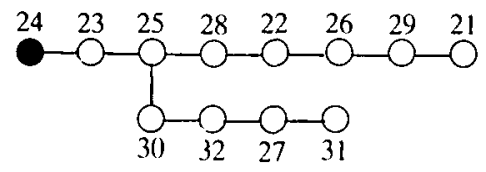

In the previous construction the total number of choices for $P_{1}, P_{2}, P_{3}$ was $1 \times 2 \times 2=4$, leading to four distinct cube roots of $E$ (although the reader should be aware that different choices of the maximal paths $P_{i}$ does not automatically lead to different roots; e.g. see the example of [4]). It is also conceivable that $\left(T_{1}, T_{3}, T_{2}\right)$ might be an offspring triple, but this is not the case, for then the triple of subtrees $\left(T_{1}^{\prime}, T_{2}^{\prime}, T_{3}^{\prime}\right)=\left(\mathrm{O}_{1}^{31}\right.$ $\left.{ }^{27}-25,{ }^{32}{ }^{23}\right)$ which results is not an offspring triple $(p=3, r=0, j=1, k=1)$ as $T_{2}^{\prime}$ is positioned at $r+1=1$ instead of the required position of $r=0$.

Therefore the total number of cube roots of $\alpha$ is $1 \times 4 \times 4=16$. The one corresponding to our detailed calculation is:
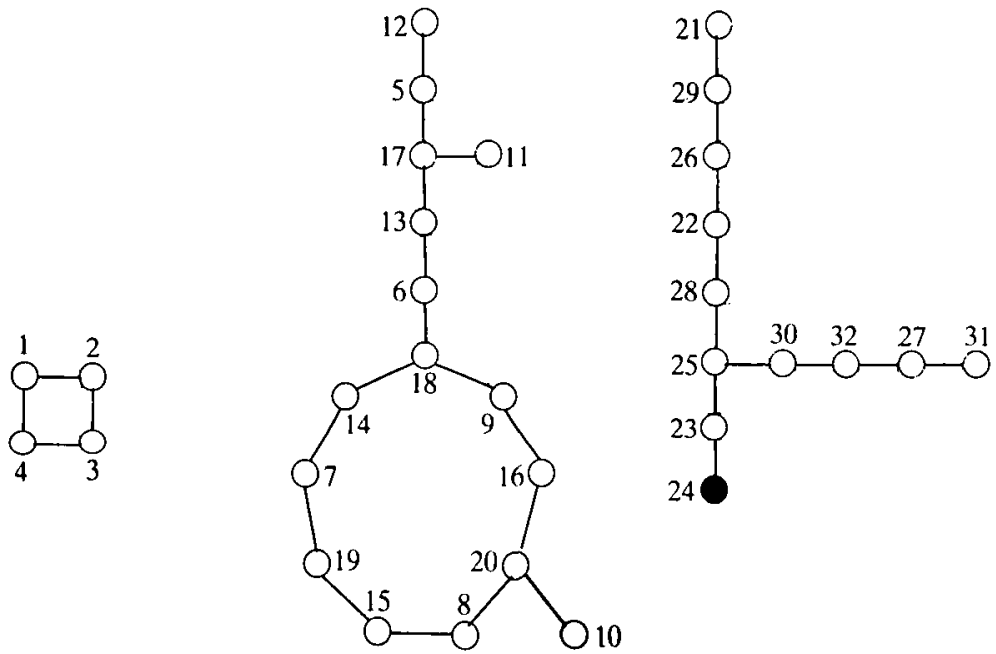

As another example we find all the 6th roots of the idempotent $\alpha \in T_{4}$

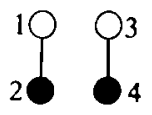

The full details are left to the reader, with the reminder that offspring tuples involving one-arrow trees may include trivial trees with no non-root points. The square roots of $\alpha$ 
are:

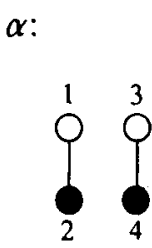

$\beta$ :
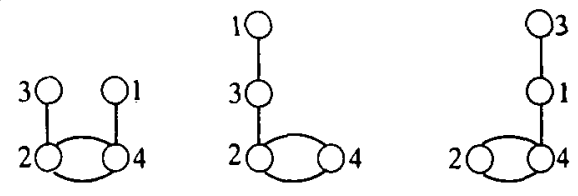

the two unnamed square roots are clearly not cubes. The sixth roots of $\alpha$ are then the cube roots of $\alpha$ and $\beta$, which are:
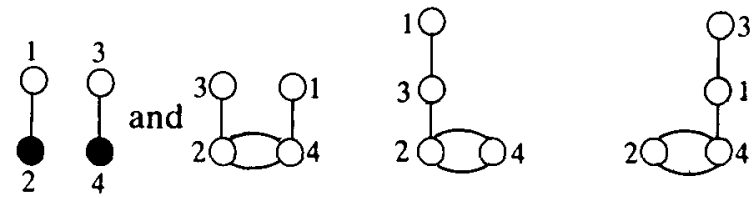

respectively.

For a given $a \in T_{n}$ the subset $C_{a}=\{b \mid a b=b a\}$, which consists of all members of $T_{n}$ which commute with $a$, is a subsemigroup of $T_{n}\left(C_{a}\right.$ is non-empty as it includes all powers of $a$ ). The determination of $C_{a}$ is equivalent to the solution of the equation $a x=x a$. We next provide a method of solution for the more general equation $a x=x b\left(a, b \in T_{n}\right)$.

Theorem 5. Given $a, b \in T_{n}$ construct $x \in T_{n}$ (if possible) as follows. For each component $A$ of a choose a component $B$ of $b$ such that $o(B) \mid o(A)$. Take $r_{0} \in V(A)$ such that $d\left(r_{0}\right)$ is maximum. Choose $\bar{r}_{0} \in V(B)$ such that $d\left(\bar{r}_{0}\right) \leq d\left(r_{0}\right)$. Then for all $k \geq 0$ put $r_{0} a^{k} x=\bar{r}_{0} b^{k}$ (we interpret $a^{0}, b^{0}$ as the identity map on $\bar{n}$ ). Next carry out the following procedure until $s x$ is defined for all $s \in V(A) .\left(^{*}\right)$ Choose $s \in V(A)$ such that $d(s)$ is maximum amongst all $s \in V(A)$ with $s x$ not defined. Let $k$ be the least positive integer such that sa $a^{k}$ is defined. Choose a directed path $s_{0} \rightarrow s_{1} \rightarrow \ldots \rightarrow s_{k-1} \rightarrow s_{k}=s a^{k} x$ in B. Define $s a^{t} x=s_{t}(0 \leq t \leq k)$.

The map $x \in T_{n}$ so constructed is a solution of $a x=x b$ and all solutions to this equation arise from this procedure.

Proof. Let $k, l$ be non-negative integers and suppose $r_{0} a^{k}=r_{0} a^{k+l}$. Then $r_{0} a^{k} \in Z_{A}$, and thus $\bar{r}_{0} b^{k} \in Z_{B}$ since $d\left(\bar{r}_{0}\right) \leq d\left(r_{0}\right)$. Now $o(B)|o(A)| l$, whence $\bar{r}_{0} b^{k+l}=\bar{r}_{0} b^{k}$; thus the rule for $x$ is well-defined, at least for all the images of $r_{0}$ under $a$. Observe that each point of $Z_{A}$ is a member of $\left\{r_{0} a^{k} \mid k \geq 0\right\}$. Furthermore, $\left(r_{0} a^{k}\right) a x=r_{0} a^{k+1} x=\bar{r}_{0} b^{k+1}=\bar{r}_{0} b^{k} \cdot b=$ $\left(r_{0} a^{k}\right) x b$, whence $a x$ and $x b$ agree on $r_{0} a^{k}$ for all $k \geq 0$.

Now suppose that the procedure $\left({ }^{*}\right)$ has been carried out $i \geq 0$ times, and that for all members $s$ of $V(A)$ for which $s x$ has been assigned, $s x$ is well-defined and $s a x=s x b$. Denote the members of $V(A)$ chosen during the procedure $\left({ }^{*}\right)$ at each stage by $r_{0}$, $r_{1}, \ldots, r_{i}$. Procedure $\left({ }^{*}\right)$ is now repeated with choice $r_{i+1}$. Let $k$ be the least positive integer such that $r_{i+1} a^{k} x$ is defined. Since $r_{i+1} a^{t} \notin Z_{A}$ for all $t<k$, it follows that 
$d\left(r_{i+1}\right)=k+d\left(r_{i+1} a^{k}\right)$. Let $r_{j}(0 \leq j \leq i)$ be such that $r_{i+1} a^{k}=r_{j} a^{t}$ for some integer $t$. Then $t \geq k$, as otherwise we would have

$$
d\left(r_{j}\right) \leq d\left(r_{j}, r_{i+1} a^{k}\right)+d\left(r_{i+1} a^{k}\right)=t+d\left(r_{i+1} a^{k}\right)<k+d\left(r_{i+1} a^{k}\right)=d\left(r_{i+1}\right),
$$

which contradicts the maximality condition on $d\left(r_{j}\right)$. A required directed path in $B$ is given by

$$
r_{j} a^{t-k} x \rightarrow r_{j} a^{i-k+1} x \rightarrow \ldots \rightarrow r_{j} a^{t} x
$$

which we write as

$$
s_{0} \rightarrow s_{1} \rightarrow \ldots \rightarrow s_{k}=r_{i+1} a^{k} x .
$$

Define $r_{i+1} a^{p} x=s_{p}(0 \leq p \leq k)$. Clearly this is well defined and furthermore, for $0 \leq p \leq$ $k-1$,

$$
\begin{aligned}
r_{i+1} a^{p} a x=r_{i+1} a^{p+1} x=s_{p+1}=r_{j} a^{t-k+p+1} x= & r_{j} a^{t-k+p} a x \\
& =r_{j} a^{t-k+p} x b \text { (by induction) }=s_{p} x b=r_{i+1} a^{p} x b
\end{aligned}
$$

as required. Since the above procedure must terminate at some stage, this completes the direct half of the proof.

Conversely, suppose that $a x=x b\left(a, b, x \in T_{n}\right)$. Suppose that $i, j \in \bar{n}$ are such that $i a^{k}=j a^{l}$ for some $k, l \geq 0$. Then $i x b^{k}=i a^{k} x=j a^{l} x=j x b^{l}$, whence $i x$ and $j x$ are in the same component of $b$. Hence for each component $A$ of $a, x$ defines a map, which we also call $x: V(A) \rightarrow V(B)$, for some component $B$ of $b$. Next suppose that $i \in Z_{A}$ and that $o(A)=k$. Then, as above, we get $i=i a^{k}$, whence $i x=i x b^{k}$, from which it follows that the restriction mapping $x \mid Z_{A}$ maps into $Z_{B}$ and moreover $o(B) \mid O(A)$.

Next let $r_{0} \in V(A)$ be such that $d\left(r_{0}\right)$ is maximum. Then $d\left(r_{0} x\right) \leq d\left(r_{0}\right)$, for if $d\left(r_{0}\right)=p<d\left(r_{0} x\right)$ we would have $r_{0} a^{p} \in Z_{A}$, but $r_{0} a^{p} x=r_{0} x b^{p} \notin Z_{B}$, contrary to what has been proved. Hence the mapping $\bar{x}: V(A) \rightarrow V(B)$ which sends $r_{0}$ to $r_{0} x$ is consistent with the initial procedure of the algorithm, and agrees with $x$ on $\left\{r_{0} a^{t} \mid t \geq 0\right\}$. Suppose inductively that we have repeated the procedure $\left(^{*}\right) i \geq 0$ times, and that the values assigned to the map $\bar{x}: V(A) \rightarrow V(B)$ that is being constructed so far agree with the map $x: V(A) \rightarrow V(B)$. We repeat the procedure and begin by choosing a suitable $r_{i+1}$. Let $k$ be the least positive integer such that $r_{i+1} a^{k} \bar{x}=r_{i+1} a^{k} x$ is defined. Denote $r_{i+1} a^{i} x$ by $s_{t}(0 \leq t \leq k)$. Then $s_{0} \rightarrow s_{1} \rightarrow \ldots \rightarrow s_{k}$ is a directed path in $B$, as for all $0 \leq t \leq k-1$,

$$
s_{t} b=r_{i+1} a^{t} x b=r_{i+1} a^{t} a x=r_{i+1} a^{t+1} x=s_{t+1} \text {, }
$$

as required. We then define $r_{i+1} a^{t} \bar{x}(0 \leq t \leq k-1)$ in accord with this path, that is $r_{i+1} a^{t} \bar{x}=r_{i+1} a^{t} x$, whereupon $x$ and $\bar{x}$ remain in agreement. This establishes that the mapping $x$ does arise out of the procedure laid down in the algorithm, thus completing the proof.

For the sake of the completeness we make a passing comment on the relatively simple equations $a x=b x$ and $x a=x b\left(a, b \in T_{n}\right)$. Note that $a x=b x$ if and only if $\{(i a, i b) \in \operatorname{ker} x$ for all $i \in \bar{n}\}$. Hence this equation defines a partition $P$ of $\bar{n}(j P k$ if there 
exists $i \in \bar{n}$ such that $j=i a, k=i b)$ and the solution set of the equation is $\{\alpha \in$ $T_{n} \mid$ ker $\left.\alpha \supseteq P\right\}$. This set forms a right ideal of $T_{n}$ containing the constant functions. The equation $x a=x b$ can be solved through consideration of the (perhaps empty) set $Y$, which is the largest subset of $\bar{n}$ such that $a|Y=b| Y$. Clearly $x a=x b$ if and only if $\nabla x \subseteq Y$. The order of the solution set is then $|Y|^{n}$ and, unless empty, this set forms a left ideal of $T_{n}$.

As an application of our Theorem 5, we determine those members $a \in T_{n}$ for which $C_{a}$ is as small as possible.

THEOREM 6. Let $a \in T_{n}$. Then $C_{a}=\left\{a^{k}: k \geq 0\right\}$ if and only if a has a unique component and a unique endpoint, or every component of $a$ is cyclic and the orders of these components are pairwise relatively prime.

Proof. Suppose that $a$ has a unique component $A$ with a unique endpoint $r$. By the algorithm of Theorem 5 we see that a member $x \in C_{a}$ is determined by $r x=r a^{k}$ for some $k \geq 0$, whereupon it is evident that $x=a^{k}$. Next suppose that all components of $a$ are cyclic, and that the orders of these components are pairwise relatively prime. Since the orders are relatively prime and $a$ has no fixed points, it follows that for any $x \in C_{a}$ and component $A$, the function $x$ maps $V(A)$ to itself. Hence if the (cyclic) components of $a$ are $A_{1}, A_{2}, \ldots, A_{k}$ say, we have that $x\left|V\left(A_{i}\right)=a^{t_{i}}\right| V\left(A_{i}\right)$, for some positive integer $t_{i}$ $(1 \leq i \leq k)$. By the Chinese Remainder Theorem, the system of congruences

$$
y \equiv t_{i}\left(\bmod o\left(A_{i}\right)\right) \quad(i=1,2, \ldots, k)
$$

has a unique solution $\bmod o\left(A_{1}\right) o\left(A_{2}\right) \ldots o\left(A_{k}\right)$, which we denote by $t$. Hence $x\left|V\left(A_{i}\right)=a^{t}\right| V\left(A_{i}\right)$, for all $i=1,2, \ldots, k$. In other words $x=a^{t}$ as required. This completes the direct half of the proof.

Conversely, suppose that $a$ has two distinct components $A$ and $B$. Suppose that $o(B)=1$ so that $V\left(Z_{B}\right)=\{p\}$ say. Define $x \in T_{n}$ by

$$
i x=\left\{\begin{array}{lll}
i & \text { if } i \notin V(A), \\
p & \text { if } & i \in V(A) .
\end{array}\right.
$$

By construction we get $a x=x a$. However $x \notin\left\{a^{k} \mid k \geq 0\right\}$ as for $i \in V(A), i a^{k} \neq p=i x$, for every $k \geq 0$. Hence if $C_{a}=\left\{a^{k} \mid k \geq 0\right\}$ and $a$ has two distinct components, then $a$ has no fixed points. Suppose that $a$ satisfies the conditions of the previous sentence but that the component $A$ of $a$ has an endpoint $r$. Define $x \in T_{n}$ by

$$
i x= \begin{cases}i & \text { if } i \notin V(B), \\ i a & \text { if } i \in V(B) .\end{cases}
$$

Again, by construction, $x \in C_{a}$, but suppose $x=a^{k}$ for some $k \geq 0$. Then since $r x=r$, and $r \notin \nabla a$, this implies $k=0$; that is $x$ is the identity. But $x|V(B) \neq 1| V(B)$, as $a$ has no fixed points.

We conclude that if $C_{a}=\left\{a^{k} \mid k \geq 0\right\}$ then any pair of distinct components $A$ and $B$ of $a$ are cyclic, of orders $m_{1}$ and $m_{2}$ say. Consider again the mapping $x \in C_{a}$ introduced in 
the preceding paragraph. If $x=a^{k}$ for some $k \geq 0$ then $k=k_{1} m_{1}=k_{2} m_{2}+1$ for some non-negative integers $k_{1}$ and $k_{2}$. But then $k_{1} m_{1}-k_{2} m_{2}=1$ which implies that $m_{1}$ and $m_{2}$ are coprime.

Finally suppose that $a$ has a unique component $A$ with two distinct endpoints $r$ and $s$ and that

$$
d(r)=\max _{u \in V(A)} d(u)
$$

Let $l$ be the least positive integer such that $s a^{l}=r a^{t}$ for some positive integer $t$. Observe that $t \geq l$ as $d(r) \geq d(s)$. Define $x \in T_{n}$ by

$$
\begin{aligned}
& i x=i \text { if } i \notin\left\{s a^{p} \mid 0 \leq p \leq l-1\right\}, \\
& s a^{p} x=r a^{t-l+p} \text { for all } 0 \leq p \leq l,
\end{aligned}
$$

observing that the definitions coincide when $p=l$. Now $x \in C_{a}$, as if $i \notin\left\{s a^{p} \mid 0 \leq p \leq\right.$ $l-1\}$ then $i x a=i a=i a x$, while $s a^{p} x a=r a^{t-l+p+1}=s a^{p+1} x=s a^{p} a x$ for all $0 \leq p \leq l-1$. Finally suppose that $x=a^{k}$ for some $k \geq 0$. Since $r x=r$, and $r \notin \nabla a$, it follows that $k=0$. But $x$ is not the identity, as $s x=r a^{t-l} \neq s$ because $s \notin \nabla a$ and $r \neq s$. This completes the proof.

The author wishes to acknowledge the support of a Deakin University Post Doctoral Research Fellowship.

\section{REFERENCES}

1. A. H. Clifford and G. B. Preston, The algebraic theory of semigroups, (Math. Surveys of the Amer. Math. Soc. 7, Vol. I, Providence R.I., 1961).

2. F. Harary, Graph theory (Addison-Wesley, 1969).

3. P. M. Higgins, A method for constructing square roots in finite full transformation semigroups, Bull. Canad. Math. Soc. 29 (1986), 344-351.

4. J. M. Howie, The subsemigroup generated by the idempotents of a full transformation semigroup, J. London Math. Soc. 41 (1966), 707-716.

5. J. M. Howie, Idempotent generators in finite full transformation semigroups, Proc. Roy. Soc. Edinburgh, Sect. A 81 (1978), 317-323.

6. M. Snowden and J. M. Howie, Square roots in finite full transformation semigroups, Glasgow Math. J. 23 (1982), 137-149.

DEAKIN UNIVERSITY

VICTORIA 3217

Australia
Present address:

Mathematics Department

Royal Melbourne Institute of Technology

Melbourne

VICTORIA 3001

AUSTRalia 